\title{
Electron Mobility Enhancement Using Ultrathin Pure Ge on Si Substrate
}

\author{
Chia Ching Yeo, Byung Jin Cho, Senior Member, IEEE, F. Gao, S. J. Lee, Member, IEEE, M. H. Lee, C.-Y. Yu, \\ C. W. Liu, L. J. Tang, and T. W. Lee
}

\begin{abstract}
We demonstrate enhancement of electron mobility in nMOSFET using an ultrathin pure Ge crystal channel layer directly grown on a bulk $\mathrm{Si}$ wafer. A thin $\mathrm{Si}$ crystal layer is also grown on top of a Ge crystal channel layer as a capping layer. Using the $\mathrm{Si} / \mathrm{Ge} / \mathrm{Si}$ structure, a maximum $2.2 \mathrm{X}$ enhancement in electron mobility is achieved while good gate dielectric properties and junction qualities of bulk Si devices are maintained.
\end{abstract}

Index Terms-Effective electron mobility, Ge, high- $K$ gate dielectric.

\section{INTRODUCTION}

$\mathbf{R}$ EPLACEMENT of $\mathrm{SiO}_{2}$ with high- $K$ gate dielectrics has highlighted new challenges such as channel mobility degradation [1], [2]. Recently, MOSFET on Ge substrate is extensively investigated as it can offer two times and four times higher electron and hole mobilities, respectively [3]. However, integration of bulk Ge into CMOS processes has encountered several critical problems, such as poor mechanical properties and limited supply of raw material. Unlike $\mathrm{Si}$, the low thermal stability of $\mathrm{Ge}$ and water-solubility of $\mathrm{GeO}$ has hindered the realization of good interfacial quality with gate dielectric [4], [5]. In addition, smaller bandgap of Ge has resulted in high source/drain junction leakage current [4], [6]. In this letter, we propose and demonstrate a simple device structure to exploit the advantage of high carrier mobility of Ge channel, while utilizing other excellent properties of Si substrate, such as good mechanical properties, excellent integrity with gate dielectric and good on-off ratio of junction current.

\section{Device Structure}

Due to $4 \%$ lattice constant mismatch and surface energy difference between $\mathrm{Ge}$ and $\mathrm{Si}$, formation of islands and three-dimensional clusters have been observed if Ge is grown directly on Si [7]. Nevertheless, growth of continuous and defect free crystal Ge layer on Si crystal is indeed still possible if the $\mathrm{Ge}$

Manuscript received July 8, 2005. The review of this letter was arranged by Editor B. Yu.

C. C. Yeo, B. J. Cho, F. Gao, and S. J. Lee are with the Silicon Nano Device Laboratory, Department of Electrical and Computer Engineering, National University of Singapore, Singapore 119260, Singapore (e-mail: elebjcho@nus.edu.sg).

M. H. Lee, C.-Y. Yu, and C. W. Liu are with the Electronics Research and Service Organization, Industrial Technology Research Institute, Chutung, Hsinchu 310, Taiwan, R.O.C.

L. J. Tang is with the Institute of Microelectronics (IME), Singapore 117685, Singapore.

T. W. Lee is with the Jusung Engineering Company, Ltd., Kyunggi-Do 464892 , Korea.

Digital Object Identifier 10.1109/LED.2005.855420
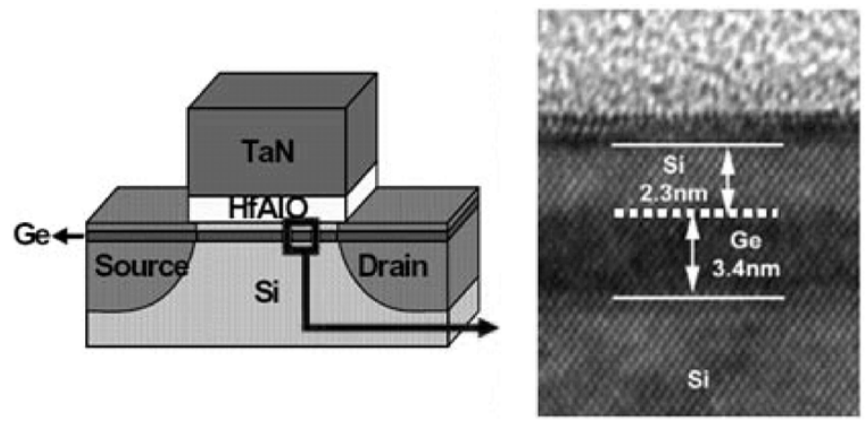

Fig. 1. (a) Schematic diagram of Si/Ge/Si (SGS) MOSFET. Majority part of the S/D is in the Si substrate and a thin Ge layer lies at the channel region. (b) XTEM image of $3.4 \mathrm{~nm}$ of Ge epitaxially grown on Si substrate, with a thin $\mathrm{Si}$ capping layer of $2.3 \mathrm{~nm}$.

layer grown is thinner than critical thickness, a thickness in which dislocations or islands starts to form when strain is relaxed. It is reported that the critical thickness of $\mathrm{Ge}$ on $\mathrm{Si}$ is below $10 \mathrm{~nm}$, depending on the process conditions [8], [9]. Quantum mechanical behavior of the inversion electrons has shown that the electron concentration peaks at a distance of 1 to $2 \mathrm{~nm}$ from the gate dielectric/Si interface, with the whole inversion layer depth extends to several $\mathrm{nm}$ into the substrate, depending on the surface electric field. For the exploitation of advantage of high channel mobility, therefore, it is not necessary for high mobility channel to be thick. Considering such, we propose to use an ultrathin crystal Ge layer directly on $\mathrm{Si}$ substrate, with its thickness thinner than critical thickness. On top of this Ge layer, we grow an additional ultrathin $\mathrm{Si}$ epi layer. MOSFETs are then fabricated on this $\mathrm{Si} / \mathrm{Ge} / \mathrm{Si}$ (SGS) substrate, as shown in Fig. 1(a). In such transistor, carrier transport can occur in the Ge layer while majority part of source/drain junction is still in Si substrate. The electron population distribution will mainly reside in the Ge layer when the $\mathrm{Si}$ capping layer is very thin $(<1 \mathrm{~nm})$. Gate dielectric is formed on top of silicon surface. Therefore, we can achieve high channel mobility of Ge devices while maintaining other properties of Si devices.

\section{DEVICE FABRICATION}

A Si buffer layer of $44 \mathrm{~nm}$ was epitaxially grown at $525^{\circ} \mathrm{C}$ on a p-type $\mathrm{Si}(100)$ substrate $(15-25 \Omega \mathrm{cm})$ by UHV/CVD method, using $\mathrm{SiH}_{4}$ as precursor. A pseudomorphic Ge layer was then epitaxially grown with $\mathrm{GeH}_{4}$ precursor, followed by growth of a thin Si capping layer. Fig. 1(b) shows the cross-sectional transmission electron microscope (TEM) image of as-deposited $\mathrm{Si} / \mathrm{Ge} / \mathrm{Si}$ (SGS) layers, with thickness of $\mathrm{Ge}$ and $\mathrm{Si}$ capping layer of 3.4 and $2.3 \mathrm{~nm}$, respectively. From the cross-sectional TEM 
image, no dislocations and defects are observed at the Ge/Si interfaces. The root mean square of surface roughness is $0.516 \mathrm{~nm}$ as measured by atomic force microscopy, indicating no sign of Ge island formation on top of Si substrate. For the MOSFET fabrication, Si capping layer thickness was further reduced just before gate dielectric deposition by repeating wet chemical oxidation and stripping process using $\mathrm{SC} 1\left(\mathrm{NH}_{4} \mathrm{OH}: \mathrm{H}_{2} \mathrm{O}_{2}\right.$ : DIW) and diluted $\mathrm{HF}$ at room temperature. Three different final Si capping layer thicknesses of $2.3 \mathrm{~nm}, 1.5 \mathrm{~nm}$, and $0.7 \mathrm{~nm}$ were prepared. HfAlO was deposited as gate dielectric at $450{ }^{\circ} \mathrm{C}$, followed by a post deposition annealing at $500{ }^{\circ} \mathrm{C}$ for $60 \mathrm{~s}$ in a $\mathrm{N}_{2}$ ambient. TaN was used as gate electrode. For source/drain (S/D) formation, a method of amorphization and solid-phase epitaxy (SPE) re-growth at a low temperature was employed. Arsenic ions were implanted with a dose of $3 \times 10^{15} \mathrm{~cm}^{-2}$ at $32 \mathrm{keV}$ and then annealed using furnace at $550{ }^{\circ} \mathrm{C}$ for $10 \mathrm{~min}$ in a $\mathrm{N}_{2}$ ambient for SPE regrowth. Finally, Al metallization process for contact and forming gas annealing were implemented.

\section{RESULTS AND DISCUSSION}

Fig. 2 shows the capacitance-voltage $(C-V)$ characteristic of SGS nMOSFET with Si capping layer thickness of $2.3 \mathrm{~nm}$. Unlike the $C-V$ curve of Ge MOS capacitors [5], no apparent $D_{\text {it }}$ generated is observed in SGS MOS capacitors. A small plateau was observed during weak accumulation $\left(V_{g}=\sim 0\right.$ $\mathrm{V})$, attributed to holes confinement in the $\mathrm{Si} / \mathrm{Ge} / \mathrm{Si}$ heterostructure, as depicted in the inset of Fig. 2. The holes confinement in this potential well effectively increases the equivalent oxide thickness (EOT) during weak accumulation. Accumulated holes only occur at the Si capping/HfAlO interface during strong accumulation $\left(V_{g}<-1 \mathrm{~V}\right)$. When the Si capping thickness is thinner, this effect becomes less prominent. Whereas during inversion $\left(V_{g}>1\right)$, electron will populate the HfAlO/Si capping interface.

Fig. 3(a) shows the leakage current density $\left(J_{g}\right)$ versus EOT, both for SGS and bulk Si nMOSFETs. The SGS nMOSFET has shown comparable leakage current to those of other high- $K$ on bulk Si devices obtained by other research groups [10], [11]. Fig. 3(b) shows the $n^{+}$S/D junction properties of the SGS nMOSFET as compared to junction properties of bulk $\mathrm{Si}$ nMOSFET and bulk Ge nMOSFET [4]. Bulk Si nMOSFET implanted with arsenic with a dose of $1 \times 10^{15} \mathrm{~cm}^{-2}$ at 100 $\mathrm{keV}$ and activated by rapid thermal anneal (RTA) at $900{ }^{\circ} \mathrm{C}$ for $30 \mathrm{sec}$ is included for comparison. As majority of the S/D area is in the $\mathrm{Si}$ substrate, the reversed leakage current for the SGS MOSFET is about three orders of magnitude lower than reported data for $n^{+} S / D$ of Ge substrate at reversed bias of $-1 \mathrm{~V}$ [4]. However, the reversed leakage current of SGS nMOSFET is more than one order of magnitude higher at -1 $\mathrm{V}$, compared to bulk Si nMOSFET with high temperature RTA, which requires further optimization of S/D activation process for SGS devices.

Fig. 4(a)-(c) shows the $I_{d}-V_{d}$ characteristics of SGS nMOSFET for different $\mathrm{Si}$ capping thickness, compared with Si nMOSFET. The SGS nMOSFET shows distinct enhancement of $19.4 \%, 35.7 \%$, and $47.5 \%$ compared to bulk $\mathrm{Si}$ nMOSFET, for the Si capping layer thickness of 2.3 to

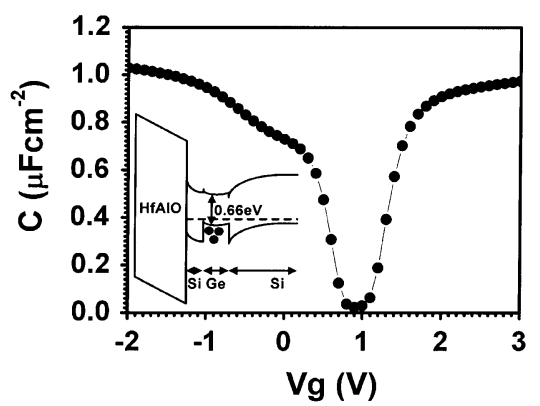

Fig. 2. $C-V$ characteristics of TaN/HfAlO for SGS MOSFET with Si capping layer thickness of $2.3 \mathrm{~nm}$. Inset shows the band diagram for SGS substrate, with Si capping layer of $2.3 \mathrm{~nm}$, indicating confined holes in the Ge valence well, resulting in thicker EOT during weak accumulation.
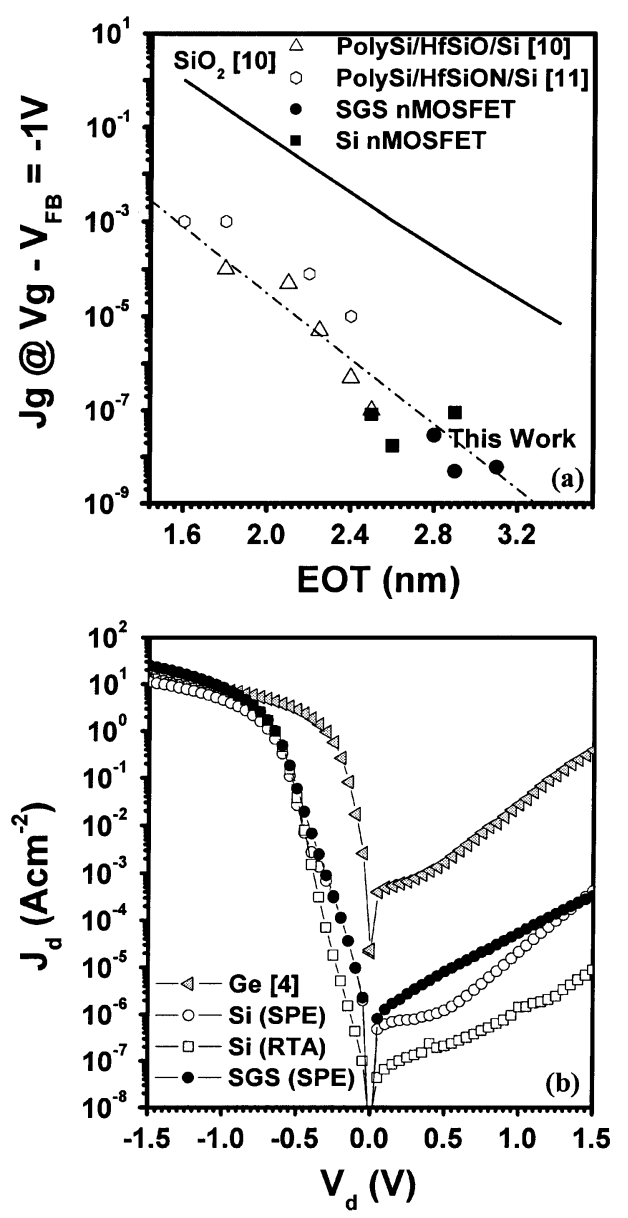

Fig. 3. (a) Gate dielectric leakage current ( $J_{g}$ at $V_{g}-V_{\mathrm{FB}}=-1 \mathrm{~V}$ ) versus EOT, showing HfAlO on SGS nMOSFET has comparable leakage current with that on bulk Si MOSFET in this letter and other reported high- $K$ on bulk $\mathrm{Si}$ [10], [11]. (b) S/D junction properties of SGS, bulk Si, and bulk Ge MOSFET [4].

1.5 and $0.7 \mathrm{~nm}$, respectively. The electron mobility in SGS nMOSFET, indicated in Fig. 4(d), measured using split $C-V$ method, also shows significant enhancement of 1.4, 1.8, and 2.2 times in peak mobility for the different Si capping layer thicknesses, respectively. The mobility enhancement depends both on the electron population distribution across the SGS structure during inversion and its straining behavior. When the electrons reside mainly in the Ge layer, as for the case of 0.7 $\mathrm{nm}$ of Si capping layer SGS nMOSFET, the channel mobility 

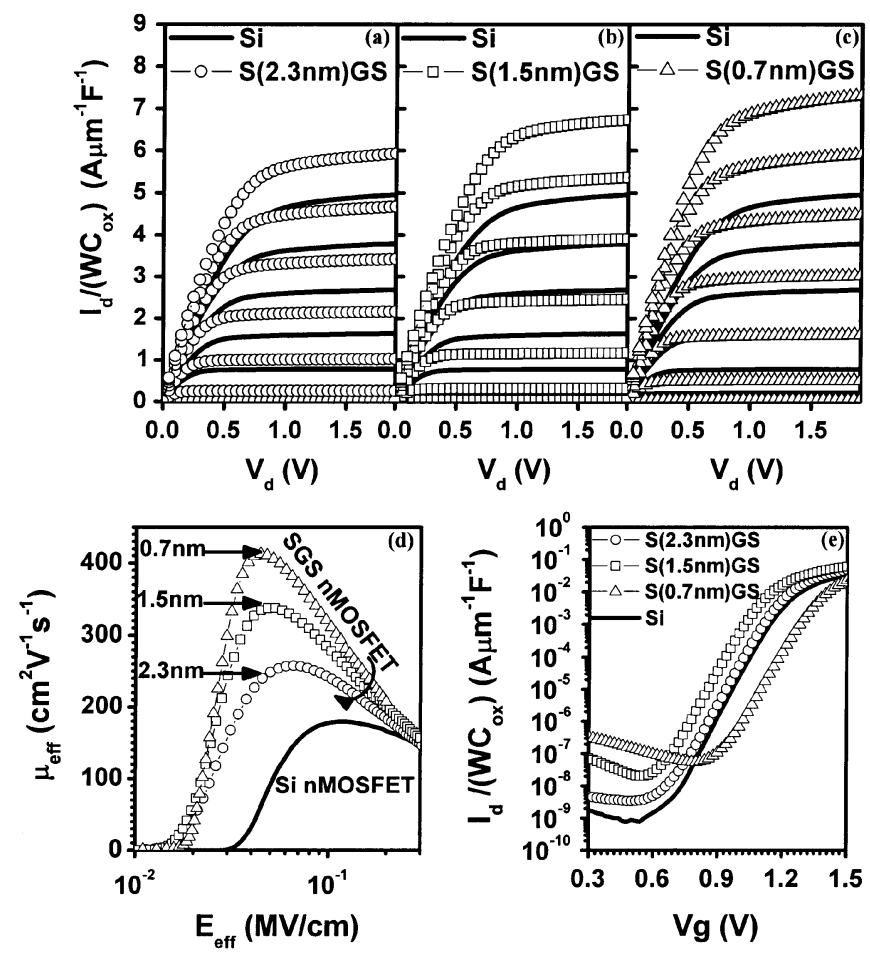

Fig. 4. $I_{d}-V_{d}$ characteristics for SGS MOSFET with Si capping layer thickness of (a) 2.3 , (b) 1.5 , and (c) $0.7 \mathrm{~nm}$. W/L of the MOSFET $=320 / 8 \mu \mathrm{m}$. Each curve is for $V_{g}-V_{T}$ of -0.2 to $1.2 \mathrm{~V}$, in increments of $0.2 \mathrm{~V}$. (d) The effective mobility versus vertical effective field for SGS and bulk Si MOSFET with HfAlO gate dielectric. (e) $I_{d}-V_{g}$ characteristics for SGS nMOSFET, as compared with Si nMOSFET.

is greatly enhanced, compared to bulk Si nMOSFET. It is well known that the electron mobility in bulk Ge is two times of that of bulk Si. In this letter, 2.2 times enhancement in electron mobility is demonstrated. The additional enhancement may be attributed to strain in the channel layer, which will be further explored later.

Fig. 4(e) compares the $I_{d}-V_{g}$ characteristics of SGS and $\mathrm{Si}$ nMOSFETs. The subthreshold swing for SGS nMOSFET with Si capping thickness of 2.3 and $1.5 \mathrm{~nm}$ is approximately 95 $\mathrm{mVdec}^{-1}$. This is relatively comparable to that of Si nMOSFET fabricated simultaneously, indicating no interface states density degradation. However, the subthreshold swing is slightly degraded to $110 \mathrm{mVdec}^{-1}$ when Si capping layer is $0.7 \mathrm{~nm}$, probably due to diffusion of Ge through the ultrathin Si to the dielectric/Si interface.

\section{CONCLUSION}

In this letter, we have demonstrated SGS nMOSFET with high- $K$ gate dielectric, showing a maximum mobility enhancement of 2.2 times than bulk Si nMOSFET. Although further optimizations on thickness combination and thermal processes need to be done, the SGS MOSFET shows a good feasibility as a new way for carrier mobility enhancement, with minimal modification to current CMOS process flow.

\section{REFERENCES}

[1] W. Zhu, J.-P. Han, and T. P. Ma, "Mobility measurement and degradation mechanisms of MOSFETs made with ultrathin high- $k$ dielectrics," IEEE Trans. Electron Devices, vol. 51, no. 1, pp. 98-100, Jan. 2004.

[2] A. L. P. Rotondaro, M. R. Visokay, A. Shanware, J. J. Chambers, and L. Colombo, "Carrier mobility in MOSFETs fabricated with Hf-Si-O-N gate dielectric, polysilicon gate electrode, and self-aligned source and drain," IEEE Electron Device Lett., vol. 23, no. 10, pp. 603-605, Oct. 2002.

[3] S. M. Sze and J. C. Irvin, "Resistivity, mobility and impurity levels in GaAs, Ge, and $\mathrm{Si}$ at $300^{\circ} \mathrm{K}$," Solid State Electron., vol. 11, pp. 599-602, 1968 .

[4] S. J. Whang, S. J. Lee, F. Gao, N. Wu, C. X. Zhu, J. S. Pan, L. J. Tang, and D. L. Kwong, "Germanium p-\& n-MOSFETs fabricated with novel surface passivation (plasma- $\mathrm{PH}_{3}$ and thin $\mathrm{AlN}$ ) and $\mathrm{TaN} / \mathrm{HfO}_{2}$ gate stack," IEDM Tech. Dig., pp. 307-310, 2004.

[5] C. O. Chui, S. Ramanathan, B. B. Triplett, P. C. McIntyre, and K. C. Saraswat, "Germanium MOS capacitors incorporating ultrathin high- $k$ gate dielectric," IEEE Electron Device Lett., vol. 23, no. 8, pp. 473-475, Aug. 2002.

[6] H. Shang, K.-L. Lee, P. Kozlowski, C. D’Emic, I. Babich, E. Sikorski, M. Ieong, H.-S. P. Wong, K. Guarini, and W. Haensch, "Self-aligned n-channel Germanium MOSFETs with a thin Ge oxynitride gate dielectric and Tungsten gate," IEEE Electron Device Lett., vol. 25, no. 3, pp. 135-137, Mar. 2004.

[7] K. R. Hoffmann, D. Reinking, M. Kammler, and M. H. Hoegen, "Surfactant grown low doped Germanium layers on Silicon with high electron mobilities," Thin Solid Films, pp. 125-130, 1998.

[8] R. C. People and J. C. Bean, "Calculation of critical layer thickness versus lattice mismatch for $\mathrm{Ge}_{\mathrm{x}} \mathrm{Si}_{1-\mathrm{x}} / \mathrm{Si}$ strained-layer heterostructures," Appl. Phys. Lett., vol. 47, pp. 322-324, 1985.

[9] Y. Fukuda, Y. Kohama, and Y. Ohmachi, "Critical thickness for the $\mathrm{Si}_{1-x} \mathrm{Ge}_{x} / \mathrm{Si}$ heterostructure," Jpn. J. Appl. Phys., vol. 29, pp. L20-L22, 1990.

[10] A. Morioka, H. Watanabe, M. Miyamura, T. Tatsumi, M. Saitoh, T. Ogura, T. Iwamoto, T. Ikarashi, Y. Saito, Y. Okada, H. Watanabe, Y. Mochiduki, and T. Mogami, "High mobility MISFET with low trapped charge in HfSiO films," in Symp. VLSI Tech. Dig., 2003, pp. 165-166.

[11] T. Watanabe, M. Takayanagi, R. Iijima, K. Ishimaru, H. Ishiuchi, and T. Tsunashima, "Design guideline of HfSiON gate dielectrics for $65 \mathrm{~nm}$ CMOS generation,” in Symp. VLSI Tech. Dig., 2003, pp. 19-20. 